\title{
How Oxytocin Receptor (OXTR) Single Nucleotide Polymorphisms Act on Prosociality: The Mediation Role of Moral Evaluation
}

\author{
Siyuan Shang ${ }^{1}$, Nan $W u^{2}$ and Yanjie Su${ }^{1 *}$ \\ ${ }^{1}$ School of Psychological and Cognitive Sciences and Beijing Key Laboratory of Behavior and Mental Health, Peking \\ University, Beijing, China, ${ }^{2}$ Teachers' College of Beijing Union University, Beijing, China
}

\section{OPEN ACCESS}

Edited by: Kimberly J. Saudino,

Boston University, USA

Reviewed by:

Ariel Knafo-Noam,

Hebrew University of Jerusalem, Israe

Lisabeth Fisher DiLalla,

Southern Illinois University

Carbondale, USA

${ }^{*}$ Correspondence:

Yanjie Su

yjsu@pku.edu.cn

Specialty section

This article was submitted to Personality and Social Psychology,

a section of the journal

Frontiers in Psychology

Received: 02 November 2016 Accepted: 02 March 2017

Published: 21 March 2017

Citation:

Shang S, Wu N and Su Y (2017)

How Oxytocin Receptor (OXTR)

Single Nucleotide Polymorphisms Act on Prosociality: The Mediation Role of Moral Evaluation.

Front. Psychol. 8:396.

doi: 10.3389/fpsyg.2017.00396
Prosociality is related to numerous positive outcomes, and mechanisms underlying individual differences in prosociality have been widely discussed. Recently, research has found converging evidence on the influence of the oxytocin receptor (OXTR) gene on prosociality. Meanwhile, moral reasoning, a key precursor for social behavior, has also been associated with variability in OXTR gene, thus the relationship between OXTR and prosociality is assumed to be mediated by moral evaluation. The current study examines the relationship in question, and includes gender as a potential moderator. Self-reported prosociality on Prosocial Tendencies Measure and evaluation on the moral acceptability of behaviors in stories from 790 Chinese adolescents (32.4\% boys) were analyzed for the influence of their OXTR single nucleotide polymorphisms (SNPS). Results showed that SNP at site rs2254298 was indirectly associated with prosocial behaviors via moral evaluation of behaviors, and this effect was moderated by gender. Our findings suggest an indirect association between genetic variations in OXTR and prosociality through moral evaluation, indicating the potential pathway from genetic variability to prosociality through level of moral development. We also provide some evidence that the role of oxytocin system may to some extent depend on gender. These findings may promote our understanding of the genetic and biological roots of prosociality and morality.

Keywords: moral evaluation, prosociality, oxytocin receptor gene, gender differences, mediation

\section{INTRODUCTION}

Prosociality is an umbrella term for positive emotions, attitudes, and behaviors directed toward others (Israel et al., 2015), which lead to known beneficial outcomes, including higher self-esteem, and positive social interactions (Christ et al., 2016). Attempts to better understand mechanisms underlying individual differences in prosociality have resulted in a large body of literature (Penner et al., 2005). Recently, genetic factors have been showed to contribute to such differences (Knafo et al., 2008; Christ et al., 2016), and multiple facets of prosociality had also been shown to be associated with certain common genetic determinant (Knafo-Noam et al., 2015). One factor of particular interest, the oxytocin receptor (OXTR) gene at the region of chromosome 3p25, which encodes the oxytocin receptor, may partly explain individual differences in social behaviors (Christ et al., 2016) and associated traits such as trust (Krueger et al., 2012). Studies show that a single nucleotide polymorphism (SNP) of this gene at rs2254298, a site in the third intron in the sequence, 
has impact on neuroanatomical correlates of social cognition in humans and subsequently on behavior (Brüne, 2012). For instance, Israel et al. (2009) found that in males the genetic variability at rs2254298 was associated with their performance in the Dictator Game, although the exact pattern was not mentioned. Parker et al. (2014) found that carriers of A allele at rs2254298 (AA, AG) scored worse on social functioning than those with two $G$ alleles. Consistent with these findings, carriers of A allele at rs2254298 among Chinese (Wu et al., 2005) and Japanese individuals (Liu et al., 2010), and of G allele among Caucasian individuals (Jacob et al., 2007) were found to have a higher risk for autism, of which social impairment was a core symptom (Quintana et al., 2015). These findings indicated the influence of OXTR gene on many facets associated with prosociality. Research addressing underlying mechanisms has found that the influence on behavioral outcomes is possibly mediated by social cognition. Christ et al. (2016) found that variabilities in OXTR SNPs, including rs2254298 and rs2268498, the latter being a site in the promoter region, were indirectly associated with prosocial behaviors via empathic tendency. However, empathic tendency might not be the only intermediate and more work is needed to show how the OXTR gene affects prosocial behavior.

As mentioned above, to study sociocognitive factors that have more immediate influence on behavior might help understand how gene-level factors work. In particular, moral reasoning had been identified as a key precursor for prosocial behavior in both theoretical and empirical studies (Eisenberg et al., 2005; Hawley and Geldhof, 2012). The relationship between moral judgment and morality-related social behavior had been identified by meta-analysis ( $\mathrm{Wu}$ and Liu, 2014), and fundamental studies of brain-behavior relationship also clarifies the mechanisms that link moral evaluation to prosocial behavior (Cowell and Decety, 2015). Longitudinal study also showed that moral reasoning predicted prosocial behaviors (Carlo et al., 2010). At the genetic end, the heritability of moral development has also been demonstrated by twin studies (Campbell et al., 2009). Moreover, studies have begun to show that OXTR polymorphisms predict individual difference in moral reasoning (Walter et al., 2012; Ohtsubo et al., 2014). Walter et al. (2012) investigated the relationship between OXTR gene and moral evaluation of social behavior, and found a significant difference between carriers and non-carriers of $\mathrm{C}$ allele at rs2268498 in the extent to which they exculpated agents for accidental harms, indicating that carriers of the $\mathrm{C}$ allele rated accidentally committed harm as significantly more blameworthy than non-carriers. These findings, together with evidence noticed above, suggested moral cognition and prosocial behavior probably share the same genetic and biological basis, and indicated moral evaluation of social behavior might mediate the association between OXTR gene and prosocial behavior. However, evidence has yet to be provided for this mediation.

In addition, the basal oxytocin level might differ in males and females (Ozsoy et al., 2009), whilst OXTR gene transcription and oxytocin receptor binding are regulated by sex hormones in animal models (Gimpl and Fahrenholz, 2001). Accordingly, there is substantial evidence suggesting that at least some of oxytocin receptor gene's influences are gender-specific. For instance, some previous studies have found associations between certain OXTR SNPs and prosocial behaviors (Israel et al., 2009), prosocial tendency (Christ et al., 2016), or autism-associated traits (Chen and Johnson, 2012) exclusively for males, whereas some other SNPs have been linked to prosociality in females (Israel et al., 2009). Similarly, after exogenous oxytocin administration, males tended to more strongly endorse self-benefit outcomes in moral dilemmas, but females were influenced in the opposite way (Scheele et al., 2014). Thus, it is reasonable to hypothesize that gender might moderate the potential pathway from OXTR gene to moral evaluation and prosocial behavior.

Thus, the present study was designed to extend previous research and bridge the gap between the variability in OXTR gene and prosocial behavior, by examining the potential mediating role of moral evaluation on the association between OXTR gene and prosocial behavior. SNP rs2254298 was of primary interest as a genetic determinant to human social cognition and behavior (Brüne, 2012), and rs2268498 was to date the only OXTR SNP known to be linked with moral development (Walter et al., 2012). Thus, these two candidate SNPs were selected to exam this potential pathway. Finally, we included gender as a potential moderator for well-established genderspecific influence of OXTR gene on moral evaluation and social behavior.

\section{MATERIALS AND METHODS}

\section{Participants}

A total of 790 Chinese adolescents (256 males, 534 females) took part in our study. All participants $\left(M_{\text {age }}=16.54\right.$ years, $S D_{\text {age }}=0.71$ years) were students in a public high school in Sichuan province, non-clinical and genetically unrelated. For some unidentified reason, in most classes of the school, the majority of students were female, which is reflected in the gender composition of the resulting sample. However, the male subsample itself is large enough for further analysis and can be fairly representative. The present experiment was approved by the Institutional Review Board of the Department of Psychology at Peking University, and written consent was obtained from each participant and, because they were minors, from their school prior to the experiment. Participants first completed a moral story task and 3 months later they completed a paper-andpencil version of the Prosocial Tendencies Measure (PTM) and had their buccal cells collected for genotyping. Each participant received a small gift upon completion.

\section{Moral Evaluation}

Moral evaluation, an index of moral development, was measured with a moral story task, an applications of theory of prosocial reasoning (Eisenberg et al., 2001). A participant was presented with four pairs of realistic stories. In each pair, the protagonist just encountered a situation where he or she was in a conflict of interest with someone else and had to make a choice regarding morality. One story would end up with the protagonist choosing to commit a moral transgression, namely "Harming others," 
"Stealing," "Refusing to Help," or "Refusing to Share," and the other would end up with an alternative, moral choice. The stealing-or-not scenarios were adapted from previous studies (Keller et al., 2003). Participants were asked to evaluate whether the protagonist should do so on a seven-point Likert scale ranging from 0 (extremely wrong) to 6 (perfectly $\mathrm{OK}$ ), and the four stories in which the protagonist did immorally were reverse scored. The internal reliability for this measure was good, with Cronbach's alpha of 0.72 .

\section{Prosocial Tendency Measure}

The PTM (Carlo et al., 2003) is a 25-item version composed of the following six subscales: public (four items), anonymous (five items), dire (three items), emotional (four items), compliant (five items), and altruistic (four items). The participants were requested to rate, on a five-point scale ranging from 1 (does not describe me at all) to 5 (describes me greatly), the extent to which the statements described themselves. Previous research has demonstrated that the Chinese version of the PTM has adequate internal reliability and validity (Kou et al., 2007). In this study, the total score of PTM was used to assess prosocial behavioral tendencies in varying situations, and the internal reliability for this measure was good, with Cronbach's alpha of 0.90 .

\section{Genotyping}

DNA was extracted from buccal cells collected by FlexiGene DNA Kit (Qiagen, Valencia, CA, USA). Two SNPs in OXTR gene (rs2268498 and rs2254298) were chosen in the Hapmap database. Both SNPs were genotyped using an improved multiplex ligation detection reaction (LDR) technique developed by Genesky Biotechnologies, Inc. (Shanghai, China), in which a multiplex PCR-ligase detection reaction method was used. Different SNPs were distinguished by different extended lengths at the $3^{\prime}$ end, and the alleles in each SNP were distinguished by different fluorescent labels of allele-specific oligonucleotide probe pairs. All primers, probes and labeling oligos were designed by and ordered from Genesky Biotechnologies, Inc. (Shanghai, China). The raw data was analyzed by GeneMapper 4.1. A random DNA sample from $5 \%$ of participants was genotyped twice for quality control, and yielded a reproducibility of $100 \%$. Table 1 summarizes the minor allele frequencies (mAFs) and $p$-values for the Hardy-Weinberg equilibrium tests. All allele frequencies were in Hardy-Weinberg Equilibrium and showed low linkage disequilibrium $\left(r^{2}=0.094\right.$; $\left.D^{\prime}=0.72\right)$.

\section{Statistical Analysis}

The sample was tested for Hardy-Weinberg equilibrium with MS-Excel. The following work was done with SPSS 22.0 for windows. Descriptive statistics for prosocial tendency and moral evaluation by genotype and gender were calculated. Analyses of variance (ANOVAs) were run to show the effects of gender, the genotype of OXTR SNPs (three types for each site), and their interaction on prosocial tendency and moral evaluation. Finally, the mediating role of moral evaluation was tested for with the PROCESS plug-in. Since there are three different genotypes on each SNP, we recoded each SNP into two dummy variables (at rs2268498, 1/0 for the presence/absence of $\mathrm{C}$ or $\mathrm{T}$ each; at rs2254298, the same codes with $\mathrm{A}$ or $\mathrm{G}$ ) and tested their association with prosocial tendency through moral evaluation, using linear regression analysis. And for gender, males were coded as 1 and females were coded as 0 . Sobel's test and bootstrapping method had been used to examine the significance of mediation effect. As noted above, two types of behavior - moral and immoral - were evaluated, but the two subsets of data yielded a similar pattern of results with respect to the role of moral evaluation. Thus, a combined score of moral evaluation for each participant was used. Separate analyses of evaluation of moral and immoral behavior are presented in the Supplementary Material.

\section{RESULTS}

The average evaluation of morality issues was $4.79(S D=0.77$; range $=0.00-6.00)$, with higher scores representing a more prosocial pattern of judgment, by which moral behavior was judged more affirmatively and immoral behavior more harshly. The average score of prosocial tendency was 3.57 ( $S D=0.47$; range $=1.00-5.00)$, with higher scores representing a stronger tendency (see Table 2). Moral evaluation of behaviors is positively related to prosocial tendency $(r=0.341, p<0.001)$.

First, the effects of two SNPs in the OXTR gene, gender, and their interactions on prosocial tendency and moral evaluation were analyzed with ANOVAs and are shown in Table 3. For rs2268498, there was a significant main effect of genotype on moral evaluation, $F(2,784)=3.27, p=0.038, \eta_{\mathrm{p}}^{2}=0.008$; follow-up pairwise comparisons showed that individuals with CT genotype judged morality issues significantly more prosocially than those with CC genotype (Cohen's $d=0.238$ ). For rs2254298, there was a significant interaction between gender and genotype on prosocial tendency, $F(2,784)=6.67, p=0.001, \eta_{\mathrm{p}}^{2}=0.017$, and another on moral evaluation, $F(2,784)=7.50, p=0.001$, $\eta_{\mathrm{p}}^{2}=0.019$. Simple effect tests showed that only in males did carriers of these three genotypes differ in prosocial tendency [Bonferroni test, $\left.F(2,784)=5.42, p=0.005, \eta_{\mathrm{p}}^{2}=0.014\right]$ and moral evaluation [Bonferroni test, $F(2,784)=5.81, p=0.003$, $\left.\eta_{\mathrm{p}}^{2}=0.015\right]$. Specifically, male G allele carriers (GA and GG) showed more prosocial tendency (GA vs. AA: $p=0.003$, Cohen's $d=0.653$; GG vs. AA: $p=0.015$, Cohen's $d=0.534$ ) and judged morality issues more prosocially as well (GA vs. AA: $p=0.002$, Cohen's $d=0.671$; GG vs. AA: $p=0.026$, Cohen's $d=0.477$ ). There was no interaction between the two SNPs on either dependent variable.

Then, multiple linear regression was used to investigate whether moral evaluation mediated the significant influence of gender, OXTR SNP (rs2254298) and their interaction on prosocial behaviors. The results were in line with the ANOVA results (see Table 4). Significant interactions between gender and the presence of $G$ allele were found on both prosocial tendency and moral evaluation, and females gave higher moral evaluation. In line with ANOVA results, the interactions between gender and genotype on the two measures showed that only in males could genotype be a significant predictor for each measure. In the model on prosociality concerning the hypothesized mediator role of moral evaluation, interaction between rs2254298 genotype 
TABLE 1 | Oxytocin receptor (OXTR) SNP genotype frequency.

\begin{tabular}{|c|c|c|c|c|c|c|}
\hline SNP & Genotype & mAF & Frequency & $n$ & Total & $p$-HWE \\
\hline rs2268498 & CC/CT/TT & $C=0.301$ & $0.08 / 0.44 / 0.48$ & $66 / 344 / 380$ & 790 & 0.335 \\
\hline
\end{tabular}

TABLE 2 | Prosocial tendency and moral evaluation by genotype and gender.

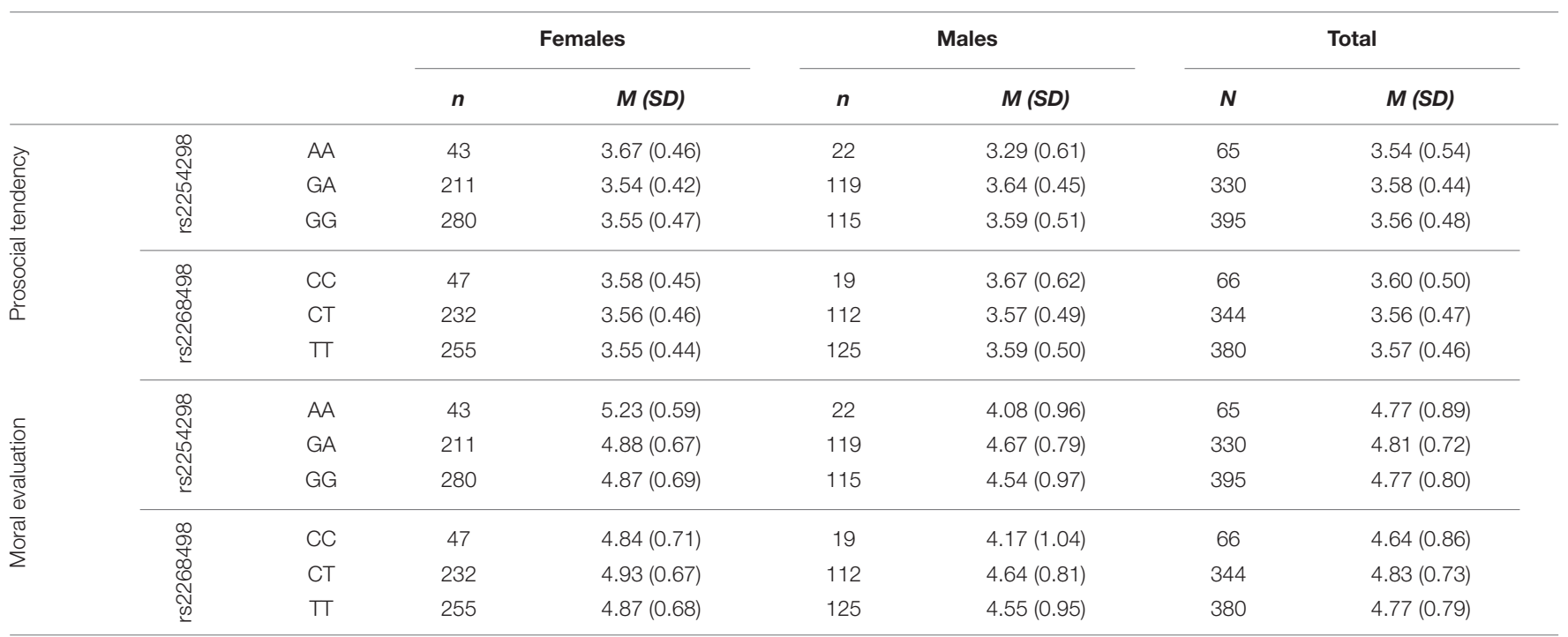

TABLE 3 | Analysis of variance (ANOVA) results for the interaction of genotype and gender on prosocial tendency and moral evaluation.

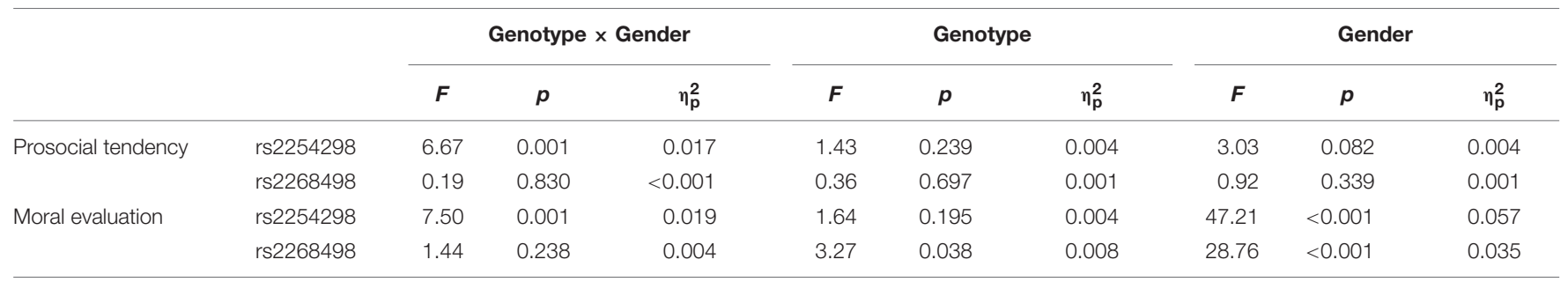

TABLE 4 | Regression analysis with gender, rs2254298A, rs2254298G, and moral evaluation predicting prosocial tendency.

\begin{tabular}{|c|c|c|c|c|c|c|}
\hline & \multicolumn{3}{|c|}{ Moral evaluation } & \multicolumn{3}{|c|}{ Prosocial tendency } \\
\hline Gender & -0.20 & $-5.78^{* * *}$ & & 0.03 & 0.84 & \\
\hline Step 2 & & & 0.001 & & & $<0.001$ \\
\hline rs2254298A & 0.04 & 1.04 & & 0.01 & 0.32 & \\
\hline rs2254298A $\times$ gender & 0.03 & 0.95 & & 0.03 & 0.83 & \\
\hline rs2254298G $\times$ gender & 0.14 & $3.87^{* * *}$ & & 0.14 & $3.65^{* * *}$ & \\
\hline Step 4 & & & - & & & $0.114^{* * *}$ \\
\hline Moral evaluation & - & - & & 0.35 & $10.16^{* * *}$ & \\
\hline
\end{tabular}

$* * p<0.01, * * * p<0.001$

and gender on prosocial tendency was reduced when moral evaluation was included, Sobel's $Z=3.58, p<0.001$. This indicated a mediating effect via moral evaluation on prosocial tendency, which had also been proved by bootstrapping method
(Preacher and Hayes, 2008), 95\% CI of coefficient $=[0.01,0.04]$. Plus, this was a partial mediation, as after the effect of moral evaluation was partialled out, the interaction between genotype and gender was still significant. 
Finally, to test whether and how gender moderated the mediation from rs2254298 genotype via moral evaluation on prosocial tendency, the mediating effect was tested by gender. The coefficients for the indirect effect via moral evaluation in both genders were significantly different from zero (95\% CI $=[0.04$, $0.26]$ for males and $[-0.10,-0.01]$ for females). However, the direction of effect varied with gender, as the plus/minus signs above implied. For males, carriers of $G$ allele at rs2254298 exhibited a more prosocial attitude in moral evaluation task and a stronger prosocial tendency than non-carriers, whereas for females, opposite patterns were observed. These mediation patterns are illustrated in Figure 1.

\section{DISCUSSION}

The present study tested the association between the variability in OXTR SNPs and both prosocial tendency and moral evaluation, and explored the mediating role of moral evaluation. The results showed that two OXTR gene SNPs tested (rs2268498 and rs2254298) were directly related to moral evaluation and the variability in OXTR SNP rs2254298 was indirectly associated with prosocial behaviors via moral evaluation of behaviors, and the latter effect was moderated by gender. These findings, coupled with previous evidence showing the relationship between OXTR gene and both prosociality (Christ et al., 2016) and moral evaluation (Walter et al., 2012), indicated prosociality and moral cognition might share similar genetic basis. Moreover, our findings suggested that moral evaluation played a mediating role in the association between OXTR gene and prosociality, indicating a genetic pathway to prosociality through moral cognition. However, as ANOVA results show, the effect sizes are relatively small, and the findings should be interpreted more cautiously.

In the present study, we found the association between OXTR rs2254298 and both moral evaluation and prosocial tendency. The differences between three genotype groups were found to be present only in male participants. The carriers of both GG and GA genotypes were found to be more prosocial and judge morality issues more prosocially than AA genotype carriers. In line with previous studies, G allele carriers were also noted to be better at perspective taking skills (Wu et al., 2012). However, we did not fully replicate the findings of Walter et al. (2012), who reported carriers of the C allele of OXTR rs2268498 judged morality issues more prosocially than noncarriers, whereas we found the carriers of the CC genotype of rs2268498 judged morality issues significantly less prosocially than the $\mathrm{T}$ allele carriers. An explanation for this incongruence lies in the demographic differences. Specifically, participants in the Walter et al. (2012) study were all Caucasian adults, whereas the participants in our study were Chinese adolescents. Previous studies have reported the difference in allele frequency of OXTR SNPs between Caucasian and Asian populations (Thompson et al., 2011). Moreover, similar population-specific findings have also been found on other SNPs. For example, rs2254298 A allele has been associated with autism spectrum disorders in Chinese (Wu et al., 2005) and Japanese populations (Liu et al., 2010), while
$\mathrm{G}$ allele is reported to be the risk allele in Caucasian populations (Jacob et al., 2007). Thus, the inconsistent findings between the two studies may be due to differential distribution and function of OXTR SNPs in the two races. It is also noteworthy that the sample in the current study was in adolescence, in which social cognitive skills undergo rapid development and the evaluation of social behaviors is of great importance in social interaction (Eisenberg et al., 2001; Steinberg and Morris, 2001). It is not clear how these developmental changes could interplay with genetic factors. Thus, more data would be needed to figure out whether the inconsistence resulted from racial or age differences, or both.

The present study also revealed effects in opposite directions of the OXTR gene (SNP rs2254298) in male and female participants. Only in male participants, G allele carriers judged morality issues significantly more prosocially and in turn tended to be more prosocial than carriers of AA genotype. By contrast, in female participants, the influence of rs2254298 was marginally significant, with $G$ allele carriers judged morality issues less prosocially and tended to be less prosocial than carriers of AA genotype. These results add to previous findings showing gender-specific influences of OXTR gene and exogenous oxytocin administration (Scheele et al., 2014; Yao et al., 2014). Specifically, Christ et al. (2016) observed an association between rs2254298 and prosocial tendency only in male participants that GG genotype was associated with more prosocial behavior. Chen and Johnson (2012) reported that A allele on rs2254298 was associated with autism-associated traits in male participants, but not in female participants. Similarly, exogenous oxytocin administration also had opposing effects on moral evaluation in males and females (Scheele et al., 2014). However, the mechanisms underlying the gender-dimorphic findings remain under debate. Some research hypothesized that such dimorphism might be associated with differences in basal oxytocin level (Ozsoy et al., 2009) and oxytocin receptor distributions (Scheele et al., 2014) in males and females, yet some other studies found no gender difference in plasma oxytocin level (Hoge et al., 2008; Feldman et al., 2012). Sex hormones might also influence the transcription of OXTR gene and regulate oxytocin receptor binding, but again, the mechanisms of regulation remain to be understood (Gimpl and Fahrenholz, 2001). Nevertheless, the current study adds some evidence for the gender-specific influence of oxytocin system, and future studies are needed to explore the underlying mechanisms.

Although the assessment of moral evaluation in the present study could not rule out the influence of implicit emotional response (Greene, 2009), and the partial mediation of moral evaluation implies the existence of other mediator(s), our finding, together with some previous evidence (Guastella et al., 2008; Andari et al., 2010; Scheele et al., 2014), suggested that oxytocin system may have its influence on prosociality partly via a cognitive mechanism. Specifically, Scheele et al. (2014) found that exogenous oxytocin administration facilitated neural responses in ACC, precuneus and insula without corresponding impact on emotional arousal, which suggested the activation in these areas might not reflect an enhanced emotional response, but 

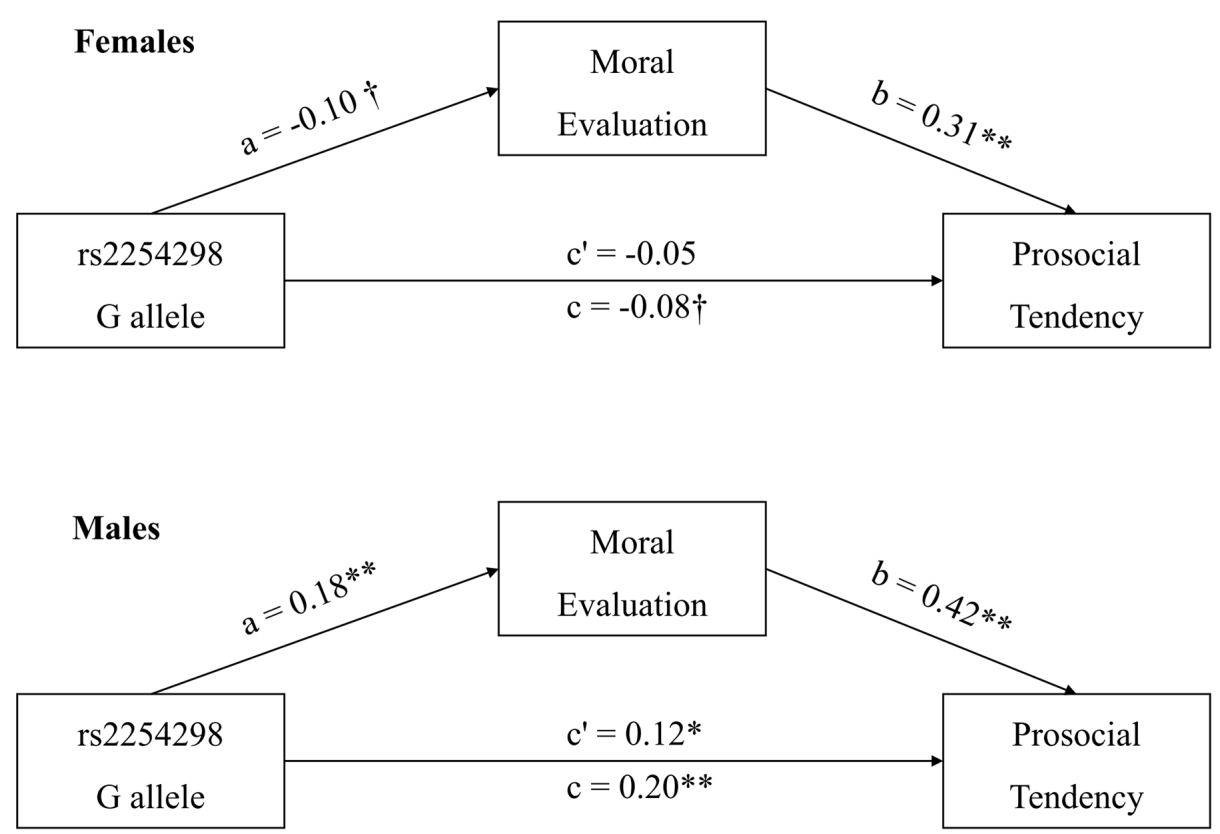

FIGURE 1 | Gender-specific effects of OXTR rs2254298 genotype on prosocial tendency mediated by moral evaluation. The mediation effects of moral evaluation in both genders were tested separately. Results showed that moral evaluation mediated the relationship between the presence of $G$ allele and prosocial tendency in both genders, but OXTR rs2254298 G allele influenced differently in males and females. ${ }^{\dagger} p<0.10,{ }^{*} p<0.05,{ }^{* *} p<0.01$.

increased attention to certain type of social cues. In line with this, exogenous oxytocin administration (Guastella et al., 2008; Andari et al., 2010) and the variability in OXTR gene (Melchers et al., 2015) have been related to social perception or attention, which can also influence moral judgment (Pärnamets et al., 2015). Additionally, at the brain activity level, OXTR gene polymorphisms have been found to be associated with the functionality of amygdala (Waller et al., 2016), which plays a pivotal role in facilitating attention to and perception of salient stimuli (Phelps and LeDoux, 2005). At the molecular level, recent research (Reuter et al., 2016) has shown that $C$ allele at rs2268498 is associated with a higher level of mRNA expression. Taken together, the literature lends support to a pathway from OXTR gene to brain function, perception, social affect, cognition, and finally to behavior. However, how the pathway operates is far from being fully understood, especially at the biological end, and further studies are needed to explore the complex mechanism underlying prosociality and morality (Israel et al., 2015).

Some limitations should be noted. First, the functionality of OXTR rs2254298 is not fully understood, especially its interaction with some other genetic variability and the environment. Thus, our study only focused on the single-gene paradigms, and future research should test multi-gene interactive effect and environmental influence as well. Second, although we had a sample large enough for a genetics study, allowing for testing the differences between three genotype groups, there are large differences in sample size between subgroups, with only 22 male participants carrying AA genotype at rs2254298. Thus, we randomly chose 22 participants from the each subgroup to conduct another ANOVA and analysis of mediation. The nature of results remained the same, as the interactions between gender and the genotype of rs2254298 on prosocial tendency and moral evaluation were still significant, and the mediation effect had also been shown with bootstrapping method. But the sample size of this randomly chosen sample is relatively small for an analysis of mediation, and becomes all the more undersized for the moderation by gender. Thus, it is essential to replicate our observations in independent samples and to extend such findings in other ethnic and age groups, which may help improve the understanding of psychosocial pathways through which individual differences in prosociality may emerge. Finally, the study was not designed to test the possibility of reciprocal influence between moral development and prosocial behaviors, which then could be a mediator on the former. More work should be done to reveal a more detailed relationship between the two measures of prosociality and morality, and their association with OXTR gene.

\section{CONCLUSION}

The present study identifies OXTR SNPs as genetic contributors to both prosociality and moral cognition, and provides evidence for the pathway from OXTR gene to moral evaluation to prosociality, as well as some more evidence for the genderspecific influence of OXTR gene. Although it is still unclear how OXTR SNPs influence prosociality and how it interacts with gender differences, this research may promote our understanding of the genetic and biological roots of prosociality and morality. 


\section{ETHICS STATEMENT}

This study was carried out in accordance with the recommendations of "Ethical Issues and Body Protection Guidelines in Psychology, Committee for Protecting Human and Animal Subjects, School of Psychological and Cognitive Sciences at Peking University"; with written informed consent from all subjects. All subjects gave written informed consent in accordance with the Declaration of Helsinki. The protocol was approved by the "Committee for Protecting Human and Animal Subjects, School of Psychological and Cognitive Sciences at Peking University."

\section{AUTHOR CONTRIBUTIONS}

SS, YS contributed to the conception and design of the work. SS collected and analyzed the data. SS, NW, YS contributed to the writing of the manuscript.

\section{REFERENCES}

Andari, E., Duhamel, J.-R., Zalla, T., Herbrecht, E., Leboyer, M., and Sirigu, A. (2010). Promoting social behavior with oxytocin in high-functioning autism spectrum disorders. Proc. Natl. Acad. Sci. U.S.A. 107, 4389-4394. doi: 10.1073/ pnas.0910249107

Brüne, M. (2012). Does the oxytocin receptor polymorphism (rs2254298) confer 'vulnerability' for psychopathology or 'differential susceptibility'? insights from evolution. BMC Med. 10:38. doi: 10.1186/1741-7015-10-38

Campbell, J., Schermer, J. A., Villani, V. C., Nguyen, B., Vickers, L., and Vernon, P. A. (2009). A behavioral genetic study of the Dark Triad of personality and moral development. Twin Res. Hum. Genet. 12, 132-136. doi: 10.1375/twin.12. 2.132

Carlo, G., Hausmann, A., Christiansen, S., and Randall, B. A. (2003). Sociocognitive and behavioral correlates of a measure of prosocial tendencies for adolescents. J. Early Adolesc. 23, 107-134. doi: 10.1177/0272431602239132

Carlo, G., Mestre, M. V., Samper, P., Tur, A., and Armenta, B. E. (2010). Feelings or cognitions? Moral cognitions and emotions as longitudinal predictors of prosocial and aggressive behaviors. Pers. Individ. Dif. 48, 872-877. doi: 10.1016/ j.paid.2010.02.010

Chen, F. S., and Johnson, S. C. (2012). An oxytocin receptor gene variant predicts attachment anxiety in females and autism-spectrum traits in males. Soc. Psychol. Pers. Sci. 3, 93-99. doi: 10.1177/1948550611410325

Christ, C. C., Carlo, G., and Stoltenberg, S. F. (2016). Oxytocin receptor (OXTR) single nucleotide polymorphisms indirectly predict prosocial behavior through perspective taking and empathic concern. J. Pers. 84, 204-213. doi: 10.1111/ jopy. 12152

Cowell, J. M., and Decety, J. (2015). The neuroscience of implicit moral evaluation and its relation to generosity in early childhood. Curr. Biol. 25, 93-97. doi: 10.1016/j.cub.2014.11.002

Eisenberg, N., Cumberland, A., Guthrie, I. K., Murphy, B. C., and Shepard, S. A. (2005). Age changes in prosocial responding and moral reasoning in adolescence and early adulthood. J. Res. Adolesc. 15, 235-260. doi: 10.1111/j. 1532-7795.2005.00095.x

Eisenberg, N., Zhou, Q., and Koller, S. (2001). Brazilian adolescents' prosocial moral judgment and behavior: relations to sympathy, perspective taking, gender-role orientation, and demographic characteristics. Child Dev. 72, 518-534. doi: 10.1111/1467-8624.00294

Feldman, R., Zagoory-Sharon, O., Weisman, O., Schneiderman, I., Gordon, I., Maoz, R., et al. (2012). Sensitive parenting is associated with plasma oxytocin and polymorphisms in the OXTR and CD38 genes. Biol. Psychiatry 72, 175-181. doi: 10.1016/j.biopsych.2011.12.025

\section{FUNDING}

This research was supported by the National Natural Science Foundation of China (Grant No 31371040, 31571134, 31170995, J1103602).

\section{ACKNOWLEDGMENTS}

We appreciate all the participants involved in this study for their enthusiastic support and participants. We appreciate our lab members who provided invaluable discussions.

\section{SUPPLEMENTARY MATERIAL}

The Supplementary Material for this article can be found online at: http://journal.frontiersin.org/article/10.3389/fpsyg. 2017.00396/full\#supplementary-material

Gimpl, G., and Fahrenholz, F. (2001). The oxytocin receptor system: structure, function, and regulation. Physiol. Rev. 81, 629-683.

Greene, J. D. (2009). Dual-process morality and the personal/impersonal distinction: a reply to McGuire, Langdon, Coltheart, and Mackenzie. J. Exp. Soc. Psychol. 45, 581-584. doi: 10.1016/j.jesp.2009.01.003

Guastella, A. J., Mitchell, P. B., and Dadds, M. R. (2008). Oxytocin increases gaze to the eye region of human faces. Biol. Psychiatry 63, 3-5. doi: 10.1016/j.biopsych. 2007.06.026

Hawley, P. H., and Geldhof, G. J. (2012). Preschoolers' social dominance, moral cognition, and moral behavior: an evolutionary perspective. J. Exp. Child Psychol. 112, 18-35. doi: 10.1016/j.jecp.2011.10.004

Hoge, E. A., Pollack, M. H., Kaufman, R. E., Zak, P. J., and Simon, N. M. (2008). Oxytocin levels in social anxiety disorder. CNS Neurosci. Ther. 14, 165-170. doi: 10.1111/j.1755-5949.2008.00051.x

Israel, S., Hasenfratz, L., and Knafo-Noam, A. (2015). The genetics of morality and prosociality. Curr. Opin. Psychol. 6, 55-59. doi: 10.1016/j.copsyc.2015.03.027

Israel, S., Lerer, E., Shalev, I., Uzefovsky, F., Riebold, M., Laiba, E., et al. (2009). The oxytocin receptor (OXTR) contributes to prosocial fund allocations in the dictator game and the social value orientations task. PLoS ONE 4:e5535. doi: 10.1371/journal.pone.0005535

Jacob, S., Brune, C. W., Carter, C. S., Leventhal, B. L., Lord, C., and Cook, E. H. Jr. (2007). Association of the oxytocin receptor gene (OXTR) in Caucasian children and adolescents with autism. Neurosci. Lett. 417, 6-9. doi: 10.1016/j. neulet.2007.02.001

Keller, M., Lourenço, O., Malti, T., and Saalbach, H. (2003). The multifaceted phenomenon of 'happy victimizers': a cross-cultural comparison of moral emotions. Br. J. Dev. Psychol. 21, 1-18. doi: 10.1348/026151003321164582

Knafo, A., Zahn-Waxler, C., Van Hulle, C., Robinson, J. L., and Rhee, S. H. (2008). The developmental origins of a disposition toward empathy: genetic and environmental contributions. Emotion 8, 737-752. doi: 10.1037/a0014179

Knafo-Noam, A., Uzefovsky, F., Israel, S., Davidov, M., and Zahn-Waxler, C. (2015). The prosocial personality and its facets: genetic and environmental architecture of mother-reported behavior of 7-year-old twins. Front. Psychol. 6:112. doi: 10.3389/fpsyg.2015.00112

Kou, Y., Hong, H., Tan, C., and Li, L. (2007). Revisioning prosocial tendencies measure for adolescent. Psychol. Dev. Educ. 23, 1112-1117. doi: 10.16187/j.cnki. issn1001-4918.2007.01.020

Krueger, F., Parasuraman, R., Iyengar, V., Thornburg, M., Weel, J., Lin, M., et al. (2012). Oxytocin receptor genetic variation promotes human trust behavior. Front. Hum. Neurosci. 6:4. doi: 10.3389/fnhum.2012.00004

Liu, X., Kawamura, Y., Shimada, T., Otowa, T., Koishi, S., Sugiyama, T., et al. (2010). Association of the oxytocin receptor (OXTR) gene polymorphisms with 
autism spectrum disorder (ASD) in the Japanese population. J. Hum. Genet. 55, 137-141. doi: 10.1038/jhg.2009.140

Melchers, M., Montag, C., Felten, A., and Reuter, M. (2015). The oxytocin receptor gene and social perception. Soc. Neurosci. 10, 345-353. doi: 10.1080/17470919. 2015.1008646

Ohtsubo, Y., Matsunaga, M., Komiya, A., Tanaka, H., Mifune, N., and Yagi, A. (2014). Oxytocin receptor gene (OXTR) polymorphism and self-punishment after an unintentional transgression. Pers. Individ. Dif. 69, 182-186. doi: 10. 1016/j.paid.2014.05.033

Ozsoy, S., Esel, E., and Kula, M. (2009). Serum oxytocin levels in patients with depression and the effects of gender and antidepressant treatment. Psychiatry Res. 169, 249-252. doi: 10.1016/j.psychres.2008.06.034

Parker, K. J., Garner, J. P., Libove, R. A., Hyde, S. A., Hornbeak, K. B., Carson, D. S., et al. (2014). Plasma oxytocin concentrations and OXTR polymorphisms predict social impairments in children with and without autism spectrum disorder. Proc. Natl. Acad. Sci. U.S.A. 111, 12258-12263. doi: 10.1073/pnas. 1402236111

Pärnamets, P., Johansson, P., Hall, L., Balkenius, C., Spivey, M. J., and Richardson, D. C. (2015). Biasing moral decisions by exploiting the dynamics of eye gaze. Proc. Natl. Acad. Sci. U.S.A. 112, 4170-4175. doi: 10.1073/pnas.1415250112

Penner, L. A., Dovidio, J. F., Piliavin, J. A., and Schroeder, D. A. (2005). Prosocial behavior: multilevel perspectives. Annu. Rev. Psychol. 56, 365-392. doi: 10.1146/ annurev.psych.56.091103.070141

Phelps, E. A., and LeDoux, J. E. (2005). Contributions of the amygdala to emotion processing: from animal models to human behavior. Neuron 48, 175-187. doi: 10.1016/j.neuron.2005.09.025

Preacher, K. J., and Hayes, A. F. (2008). Asymptotic and resampling strategies for assessing and comparing indirect effects in multiple mediator models. Behav. Res. Methods 40, 879-891. doi: 10.3758/brm.40.3.879

Quintana, D. S., Alvares, G. A., Hickie, I. B., and Guastella, A. J. (2015). Do delivery routes of intranasally administered oxytocin account for observed effects on social cognition and behavior? A two-level model. Neurosci. Biobehav. Rev. 49, 182-192. doi: 10.1016/j.neubiorev.2014.12.011

Reuter, M., Montag, C., Altmann, S., Bendlow, F., Elger, C., Kirsch, P., et al. (2016). Functional characterization of an oxytocin receptor gene variant (rs2268498) previously associated with social cognition by expression analysis in vitro and in human brain biopsy. Soc. Neurosci. doi: 10.1080/17470919.2016.1214174 [Epub ahead of print].

Scheele, D., Striepens, N., Kendrick, K. M., Schwering, C., Noelle, J., Wille, A., et al. (2014). Opposing effects of oxytocin on moral judgment in males and females. Hum. Brain Mapp. 35, 6067-6076. doi: 10.1002/hbm.22605
Steinberg, L., and Morris, A. S. (2001). Adolescent development. Annu. Rev. Psychol. 52, 83-110. doi: 10.1146/annurev.psych.52.1.83

Thompson, R. J., Parker, K. J., Hallmayer, J. F., Waugh, C. E., and Gotlib, I. H. (2011). Oxytocin receptor gene polymorphism (rs2254298) interacts with familial risk for psychopathology to predict symptoms of depression and anxiety in adolescent girls. Psychoneuroendocrinology 36, 144-147. doi: 10.1016/ j.psyneuen.2010.07.003

Waller, R., Corral-Frías, N. S., Vannucci, B., Bogdan, R., Knodt, A. R., Hariri, A. R., et al. (2016). An oxytocin receptor polymorphism predicts amygdala reactivity and antisocial behavior in men. Soc. Cogn. Affect. Neurosci. 11, 1218-1226. doi: 10.1093/scan/nsw042

Walter, N. T., Montag, C., Markett, S., Felten, A., Voigt, G., and Reuter, M. (2012). Ignorance is no excuse: moral judgments are influenced by a genetic variation on the oxytocin receptor gene. Brain Cogn. 78, 268-273. doi: 10.1016/j.bandc. 2012.01.003

$\mathrm{Wu}, \mathrm{N} ., \mathrm{Li}, \mathrm{Z}$., and Su, Y. (2012). The association between oxytocin receptor gene polymorphism (OXTR) and trait empathy. J. Affect. Disord. 138, 468-472. doi: 10.1016/j.jad.2012.01.009

Wu, P., and Liu, S. (2014). Association between moral reasoning and moral behavior: a systematic review and meta-analysis. Acta Psychol. Sin. 46, 1192-1207. doi: 10.3724/SP.J.1041.2014.01192

Wu, S., Jia, M., Ruan, Y., Liu, J., Guo, Y., Shuang, M., et al. (2005). Positive association of the oxytocin receptor gene (OXTR) with autism in the Chinese Han Population. Biol. Psychiatry 58, 74-77. doi: 10.1016/j.biopsych.2005. 03.013

Yao, S., Zhao, W., Cheng, R., Geng, Y., Luo, L., and Kendrick, K. M. (2014). Oxytocin makes females, but not males, less forgiving following betrayal of trust. Int. J. Neuropsychopharmacol. 17, 1785-1792. doi: 10.1017/ s146114571400090x

Conflict of Interest Statement: The authors declare that the research was conducted in the absence of any commercial or financial relationships that could be construed as a potential conflict of interest.

Copyright (c) 2017 Shang, Wu and Su. This is an open-access article distributed under the terms of the Creative Commons Attribution License (CC BY). The use, distribution or reproduction in other forums is permitted, provided the original author(s) or licensor are credited and that the original publication in this journal is cited, in accordance with accepted academic practice. No use, distribution or reproduction is permitted which does not comply with these terms. 